\title{
Initial seizure threshold in bilateral electroconvulsive therapy
}

\author{
Sheena R. Dykes and Allan I. F. Scott
}

\begin{abstract}
The extent to which electrical dose exceeds the seizure threshold is an important determinant of the efficacy of electroconvulsive therapy (ECT) but selzure thresholds have not before been evaluated using an ECT machine made in the UK. In this study selzure threshold was empirically fitrated at the outset of bilateral ECT given to 100 consecutive depressed patients treated with an Ectron Series 5A ECT machine. No patient recelved antiepileptic drugs. Men and women under 30 years of age $(n=14)$ had low thresholds and men of 60 years or more $(n=3)$ had high thresholds. Selzure thresholds varied of least threefold among other groups, suggesting that empirical tifration may be desirable for most patients treated by bilateral ECT.
\end{abstract}

The American Psychiatric Association (1990) recommended that the dose of electricity in electroconvulsive therapy (ECT) exceed the seizure threshold by a "moderate" amount, 50 to $200 \%$. It was accepted that further research was required to refine this recommendation. Whereas earlier guidelines from the College made no reference to the seizure threshold and advised only that the length of the convulsion be used to assess therapeutic adequacy (ECT Sub-Committee of the Research Committee, 1989), the latest guidelines recommend that the electrical dose be adjusted for each patient to take into account the variation in seizure threshold (Royal College of Psychiatrists, 1995). This technique is called 'stimulus dosing'.

There are two approaches to stimulus dosing; the empirical measurement of the seizure threshold by a technique of dose titration or the routine administration of a predetermined dose dependent on the laterality of treatment, and the age and the gender of the patient (Royal College of Psychiatrists, 1995). While the latter approach is much more straightforward, its main disadvantage is that there are few data to guide practice; moreover, findings from research centres may not be applicable to routine clinical practice. Abrams (1992) has argued that the numerical value of the seizure threshold was "entirely dependent" on the electrical characteristics of the stimulus produced by a particular ECT machine. The aim of the present study was to measure empirically initial seizure thresholds in bilateral ECT given with the Ectron Series $5 \mathrm{~A}$
ECT machine, the most widely used machine in the UK. Results were tabulated by age and gender to assess the feasibility of predicting initial seizure threshold from these variables alone.

\section{The study}

The sample comprised 100 depressed patients routinely and consecutively referred for bilateral ECT at the Royal Edinburgh Hospital. Twelve patients were not taking any concomitant psychotropic drug treatment, 29 patients took a monoamine reuptake inhibitor only, 13 a neuroleptic drug only, eight lithium carbonate only. three a monoamine-oxidase inhibitor only, three a benzodiazepine drug only and 32 took a combination of psychotropic drugs. Exclusion criteria were treatment with ECT in the previous three months and the concomitant intake of antiepileptic drugs (according to the British National Formulary).

Patients were anaesthetised with intravenous methohexitone followed by suxamethonium to induce muscle paralysis. The average dose of methohexitone was $1.2 \mathrm{mg} / \mathrm{kg}$. All patients were ventilated with about 10 inhalations of oxygen from a Boyle's circuit before electrical stimulation.

ECT was given with an Ectron Series 5A ECT machine that delivered between 50 and $700 \mathrm{mC}$ (millicoulombs) as a uniphasic brief-pulse stimulus. Seizure threshold was defined as the minimum amount of electrical charge in millicoulombs that induced a generalised tonicclonic convulsion of at least 15 seconds, which was timed by a stopwatch from the end of electrical stimulation to the end of generalised. that is bilateral, tonic-clonic muscular activity. The method of empirical titration was the Edinburgh protocol for the Ectron 5A ECT machine published in The ECT Handbook (Royal College of Psychiatrists, 1995, pp. 78-80).

\section{Findings}

The initial seizure thresholds ranged from 50 to $200 \mathrm{mC}$. Half the sample had a seizure threshold of $75 \mathrm{mC}$, the modal value, and the median seizure threshold was also $75 \mathrm{mC}$. The four patients with the lowest seizure threshold were 
two young women, one middle-aged woman and one middle-aged man. The three patients with the highest seizure threshold were two elderly women and a middle-aged woman.

The sample consisted of 78 women (average age 52 years) and 22 men (average age 45 years). Table 1 shows the relationships of seizure threshold to age and gender. There was a modest but significant correlation between age and seizure threshold for all patients $(\rho=0.35$, $P<0.001)$. The correlation was closer in men $(\rho=0.59, P<0.01)$ than in women $(\rho=0.30$, $P<0.01)$. All 14 patients who were under 30 years of age had a seizure threshold of $100 \mathrm{mC}$ or less but the variability in seizure threshold increased with age. This was particularly marked for middle-aged and elderly women when the seizure threshold ranged from $50-200 \mathrm{mC}$. The seizure threshold for middle-aged men also varied widely but the three elderly men all had high seizure thresholds.

\section{Comment}

This was the first study of seizure threshold in bilateral ECT given by the Ectron Series 5A ECT machine. Eighty per cent of patients had an initial seizure threshold between 75 and $150 \mathrm{mC}$ and half the patients had a seizure threshold of $75 \mathrm{mC}$. Only $4 \%$ of patients had an initial seizure threshold less than $50 \mathrm{mC}$ (the minimum setting on the machine) and no patient had a seizure threshold above $200 \mathrm{mC}$.

The study was limited to bilateral ECT, the electrode placement more commonly used in the UK. The initial seizure threshold in unilateral ECT would be lower (Sackeim et al, 1991). None of the sample was younger than 20 years old and further research may be desirable in young women who may have very low thresholds (Sackeim et al, 1991). There are case reports of elderly men with very high seizure thresholds (Sackeim et al, 1991) and the three old men in the present study all had initial seizure thresholds towards the upper end of the range. The present findings concern only the initial seizure threshold and this is likely to rise by an average of $80 \%$ over a course of ECT because of the anti-convulsant effect of repeated ECT (Sackeim et al, 1991).

The present findings confirm the importance of new ECT machines that can deliver a smaller dose than machines developed during the past decade. The first Ectron Series 5 ECT machine had a minimum dose of $150 \mathrm{mC}$, three times the minimum initial seizure threshold observed in the present study. The second implication of the findings concerns the desirable maximum output. Sackeim (1991) extrapolated from his research findings to suggest that the maximum output of ECT machines ought to be increased to 1000 or $1200 \mathrm{mC}$. The present findings do
Table 1. Median (range) initial seizure threshold $(\mathrm{mC})$ by age and gender

\begin{tabular}{lll}
\hline Age (years) & Male & Female \\
\hline $10-29$ & $75(75-75)$ & $75(50-100)$ \\
& $n=4$ & $n=10$ \\
$30-59$ & $125(50-175)$ & $75(50-200)$ \\
& $n=15$ & $n=31$ \\
$60-85$ & $150(150-175)$ & $100(50-200)$ \\
& $n=3$ & $n=37$ \\
\hline
\end{tabular}

not suggest that the Ectron Series 5A (maximum output $700 \mathrm{mC}$ ) is significantly underpowered. Third, debate continues about the relative merits of the two approaches to stimulus dosing (empirical measurement versus a pre-determined dose). One ECT manufacturer suggested that the initial seizure threshold can be estimated reliably from the age and gender of the patient (Swartz \& Abrams, 1994). The present findings do not suggest that age and gender alone can be used reliably to estimate the initial seizure threshold for all patients. While all patients under 30 years of age had a low seizure threshold and the elderly men all had a high seizure threshold, initial seizure threshold varied by more than threefold among the other patient groups who made up more than $80 \%$ of the total sample. The present findings suggest that empirical titration of the seizure threshold may be desirable for most patients treated by bilateral ECT.

\section{References}

ABrams, R. (1992) Electroconvulsive Therapy (2nd edn). Oxford: Oxford University Press.

AmERICAN PSYCHIATRIC Association (1990) The Practice of Electroconvulsive Therapy: Recommendations for Treatment. Training and Privileging. Washington. DC: APA.

ECT SUB-COMMITTEE OF THE RESEARCH COMMITTEE. ROYAL COLlege OF PSYCHIATRISTS (1989) The Practical Administration of Electroconvulsive Therapy (ECT) London: Gaskell.

ROYAL COLLEGE OF PSYCHIATRISTS (1995) The ECT Handbook. The Second Report of the Royal College of Psychiatrists Special Committee on ECT. Council Report CR39. London: Royal College of Psychiatrists.

SACKEIM. H. A. (1991) Are ECT devices underpowered? Convulsive Therapy. 7, 233-236.

-. Devanand. P. \& PRudic. J. (1991) Stimulus intensity. seizure threshold. seizure duration: impact on the efficacy and safety of a electroconvulsive therapy. Psychiatric Clinics of North America. 14. 803-843.

SWARTZ. C. M. \& ABRAMS. R. (1994) ECT Instruction Manual. Lake Bluff, IL: Somatics Inc.

Sheena R. Dykes, Senior Registrar, *Allan I. F. Scott. Consultant Psychiatrist and Honorary Senior Lecturer, Andrew Duncan Clinic, Royal Edinburgh Hospital, Morningside Terrace, Edinburgh EH1O 5HF

*Correspondence 